\title{
The Effect of Follow-Up Contact on Recovery After Benign Hysterectomy: A Randomized, Single-Blinded, Four-Arm, Controlled Multicenter Trial
}

Gulnara Kassymova, Gunilla Sydsjö, Ninnie Borendal Wodlin, Lena Nilsson and Preben Kjölhede

The self-archived postprint version of this journal article is available at Linköping University Institutional Repository (DiVA):

http://urn.kb.se/resolve?urn=urn:nbn:se:liu:diva-171936

N.B.: When citing this work, cite the original publication.

Kassymova, G., Sydsjö, G., Borendal Wodlin, N., Nilsson, L., Kjölhede, P., (2020), The Effect of Follow-Up Contact on Recovery After Benign Hysterectomy: A Randomized, Single-Blinded, FourArm, Controlled Multicenter Trial, Journal of Women's Health.

https://doi.org/10.1089/jwh.2020.8752

Original publication available at:

https://doi.org/10.1089/jwh.2020.8752

Copyright: Mary Ann Liebert

http://www.liebertpub.com/ 
An original article entitled

The effect of follow-up contact on recovery after benign hysterectomy - a randomized, single-blinded, four-arm, controlled multicenter trial.

Authors:

Gulnara Kassymova ${ }^{1}$, MD, (https://orcid.org/0000-0002-5386-932X), Gunilla Sydsjö ${ }^{1}, \mathrm{PhD}$, (https://orcid.org/0000-0003-4296-4038), Ninnie Borendal Wodlin ${ }^{1}, \mathrm{MD}, \mathrm{PhD}$, (https://orcid.org/0000-0002-3730-7722), Lena Nilsson ${ }^{2}$, $\mathrm{MD}, \mathrm{PhD}$, (https://orcid.org/0000-0002-7489-9077), and Preben Kjølhede ${ }^{1}$, MD, PhD, (https://orcid.org/0000-0001-5702-4116).

Affiliations:

${ }^{1}$ Department of Obstetrics and Gynecology in Linköping, and Department of Biomedical and Clinical Sciences, Linköping University, Linköping, Sweden

${ }^{2}$ Department of Anesthesiology and Intensive Care in Linköping and Department of Biomedical and Clinical Sciences, Linköping University, Linköping, Sweden

Corresponding author:

Gulnara Kassymova, MD

Department of Obstetrics and Gynecology

University Hospital

S-58245 Linköping

Sweden

Phone: $+46(0) 101030000$

E-mail: gulnara@vackerman.se

Running head line: Follow-up contact and recovery after hysterectomy 


\begin{abstract}
Background: The objective of this trial was to analyze the effect of follow-up programs using standard follow-up protocol and structured coaching on recovery after hysterectomy in an enhanced recovery after surgery setting.

Material and methods: A randomized, four-armed, single-blinded, controlled multicenter trial comprising 487 women was conducted at five hospitals in the southeast region of Sweden. The women were allocated (1:1:1:1) to either Group A: no planned follow-up contact; Group B: a single, planned, structured, broadly kept, follow-up telephone contact with the research nurse the day after discharge; Group C: planned, structured, broadly kept follow-up telephone contact with the research nurse the day after discharge and then once weekly for six weeks; and Group D: as Group C, but with planned, structured, coaching telephone contact. Recovery was assessed by the health-related quality of life (HRQoL) questionnaires EQ-5D-3L and SF36 , and duration of sick leave.
\end{abstract}

Results: Neither the recovery of HRQoL as measured by the EQ-5D-3L and the SF-36, nor the duration of sick leave (mean 26.8-28.1 days) differed significantly between the four intervention groups. Irrespective of mode of follow-up contact used, the women had recovered to their baseline EQ-5D-3L health index four weeks after surgery. The occurrence of unplanned telephone contact was significantly lower (by nearly $30 \%$ ) in the women who had structured coaching.

Conclusion: Follow-up contact including coaching did not seem to expedite the postoperative recovery in HRQoL or reduce the sick leave after hysterectomy, but the coaching seemed to reduce unplanned telephone contact with the health care services.

Keywords: Coaching; Enhanced recovery after surgery; Follow-up; Hysterectomy; Healthrelated quality of life, Recovery. 


\section{Introduction}

Hysterectomy on benign indication is the most common major gynecological operation worldwide ${ }^{1}$. The aim of surgery for benign diseases is primarily to improve the health-related quality of life (HRQoL). Recovery after surgery is therefore very important for the patient as well as for society. Enhanced recovery after surgery (ERAS) programs have been developed to facilitate recovery but are limited to pre-, intra- and postoperative care in the hospital and do not include follow-up visits or intervention after discharge ${ }^{2,3}$.

Validated follow-up strategies or treatment models of coaching to handle postoperative recovery are sparsely described ${ }^{4}$ and guidelines are lacking. Qualitative studies on patients' experience of ERAS programs point at desires to extend the program with contact from professional or experienced patient volunteers following hospital discharge to offer support and guidance ${ }^{5,6}$. Structured coaching programs have been shown to be successful for the treatment of common anxiety disorders ${ }^{7}$. Follow-up strategies using supportive coaching programs could theoretically be helpful to enhance recovery after surgery. So far, no clinical studies using such treatments or coaching have been published concerning gynecological surgery. In general, there seems to be a need to improve postoperative recovery as this has a substantial impact on the individual and on the health care system ${ }^{8,9}$.

No Swedish guidelines have been established concerning follow-up contact after hysterectomy. Today, the follow-up methods vary between telephone contact with nurses after discharge occurring at various intervals, scheduled visits to the surgeon to no planned contact. In order to evaluate the effect of the three most commonly used follow-up methods in Sweden and a follow-up strategy with structured coaching after hysterectomy we conducted a randomized, four-arm, controlled trial. The coaching program aimed to modulate the way of managing the postoperative discomfort in order to improve HRQoL. We hypothesized that women who were exposed to an oriented coaching program would experience an accelerated 
recovery in HRQoL after hysterectomy, leading to a faster return to normal daily living and fewer unplanned patient-initiated telephone contacts and visits in health care facilities compared with the women who experienced the ordinary modes of follow-up.

The primary aim of the study was to determine the effect of a structured postoperative oriented coaching program on the recovery of HRQoL in relation to other follow-up methods. The secondary aims were to assess whether the oriented coaching program also contributed to a shorter sick leave, and to evaluate the occurrence of patient-initiated unplanned telephone contact or visits to health care facilities within six weeks after surgery. In addition, the association between the HRQoL measures and duration of sick leave was analyzed. 


\section{Materials and methods}

A four-armed, semi-blinded, randomized controlled trial (RCT) of women undergoing hysterectomy for benign conditions, the Post-hysterectomy Recovery trial (POSTHYSTREC), was undertaken at the departments of obstetrics and gynecology in five public hospitals in the southeast health region of Sweden during the period October 2011 and May 2017.

Study population

All women between 18 to 60 years of age, who were admitted for abdominal or vaginal hysterectomy on benign indications, were eligible for the study. Further inclusion criteria were: being linguistically proficient in Swedish, and having access to a private telephone or the internet. Exclusion criteria were: genital prolapse as indication for the hysterectomy, previous bilateral oophorectomy or the present operation would leave the woman without ovaries, physically or mentally disabled, severe psychiatric disease, and current drug or alcohol abuse.

All clinics adapted to the perioperative ERAS program routinely in clinical practice (Supplementary Table S1). The mode of hysterectomy was chosen at the discretion of the surgeon after consultation with the patient, but had to be decided before inclusion in the study. The mode of anesthesia was chosen at the discretion of the attending anesthesiologist, but according to the ERAS program would preferably be intrathecal morphine analgesia alone or in combination with general anesthesia. The postoperative care followed the ERAS program. The study-specific demographic and clinical data were collected prospectively. Additionally, unplanned telephone contact and visits to health care facilities were registered from discharge to the planned follow-up visit six weeks after surgery. Postoperative complications were categorized according to the contracted form of the Clavien-Dindo complication classification ${ }^{10}$.

\section{Randomization}


A computer generated the randomization code ${ }^{11}$ with an allocation ratio of 1:1:1:1 of the intervention models. The allocated intervention was written on a paper enclosed in consecutively numbered sealed opaque envelopes. A block randomization was used, with allocation and stratification for abdominal or vaginal hysterectomy. After being given written and verbal information and providing written informed consent, the participants were randomized before surgery in order of the numbered envelopes. However, the randomization envelope was first opened upon discharge. All participants were informed that there would be follow-up contact with the research nurse postoperatively but the frequency of the follow-up contact was concealed, and was first revealed to the woman at the time of opening the randomization envelope at discharge. Thus, the participants were blinded to the content of the follow-up contact and also to the frequency of the follow-up contact of all other groups.

\section{Intervention models}

The four follow-up models consisted of Group A) no planned follow-up contact with the health care after discharge; Group B) one planned, structured, broadly kept, follow-up telephone contact with the research nurse the day after discharge; Group C) a planned, structured, broadly kept follow-up telephone contact with the research nurse the day after discharge and then once weekly for six weeks, and Group D) planned, structured, oriented coaching telephone contact with the research nurse the day after discharge and then once weekly for six weeks. The content of the telephone contact in Groups B and C is shown in Figure 1, and of the oriented coaching model in Group D in Figure 2.

The participants in all four groups were seen by the study nurse at the end of the study, six weeks after surgery.

During the planned telephone contact, the participants in Groups C and D were reminded to complete their study-specific forms and questionnaires. The women in Groups A and B were reminded once weekly by e-mail or SMS to their mobile phone. 


\section{Instruments to measure health-related quality of life}

The EuroQoL-5 Dimension with three levels (EQ-5D-3L) is a validated widely used generic instrument that comprises five dimensions: mobility, self-care, usual activities, pain/discomfort and anxiety/depression ${ }^{12,13}$. Each dimension has three levels of severity: no problems, some problems, extreme problems. This results in 243 combinations translated into a health index scaling from -0.594 to 1 . Zero indicates the state of death and 1 indicates good health.

The EQ-5D form was used to detect short-term changes in HRQoL and was filled in on 14 occasions: approximately one week preoperatively (baseline), then daily from the day of surgery for eight days and thereafter once a week for six weeks.

The Short-Form-Health Survey with 36 items (SF-36) is a generic widely used and robust measure of HRQoL ${ }^{14}$ and has been validated in a Swedish context ${ }^{15}$. It measures perceived health status by assessing 36 items covering eight health profiles: physical functioning, physical role limitation, bodily pain, general health perceptions, vitality, social functioning, emotional role limitation, and mental health. Sum scores are calculated and analyzed for every single profile. The eight domains can be aggregated into two psychometrically-based summary measures: the physical component summary (PCS) score and the mental component summary (MCS). The sum score for each profile ranges between 0 and 100. Higher scores indicate better HRQoL ${ }^{14,15}$.

The SF-36 was filled in approximately one week preoperatively (baseline) and at the six-week follow-up visit in order to evaluate changes in HRQoL over a longer period.

\section{Sick leave}

Sick leave is financially reimbursed by the Swedish Social Insurance Agency. The sick leave was initially granted for 14 days and thereafter, if necessary, extended repeatedly by up to seven days at a time. The woman decided when she had sufficiently recovered and could go 
back to employment. If the sick leave exceeded six weeks the woman had to see her gynecologist for evaluation in order to receive prolonged sick leave. The duration of sick leave was calculated as the number of calendar days from the day of surgery to the date of the end of sick leave as stated on the sick leave form.

\section{Outcome measures}

The primary outcome was postoperative recovery of HRQoL as measured by EQ-5D and SF36. Secondary outcome measures were duration of sick leave, frequency of unplanned telephone contacts and visits, and association between duration of sick leave and recovery of HRQoL.

\section{Ethical approval}

The study was approved by the Regional Ethics Board of Linköping University (Dnr.2011/10631, approval date 23 May; 2011). The study was conducted in accordance with the Declaration of Helsinki ${ }^{16}$, Good Clinical Practice Guidelines ${ }^{17}$, and monitored by an authorized, independent monitor. The trial was registered in ClinicalTrial.gov (NCT01526668)

\section{Statistical analysis}

The software TIBCO Statistica ${ }^{\circledR}$ 13.5.0 (TIBCO Software Inc. 3307 Hillview Avenue, Palo Alto, CA 94304 USA) was used to analyze the data.

Data are presented as mean and one standard deviation (SD) or median and range, and number and percent. Nominal data was analyzed by means of a Pearson's Chi-squared test, and continuous normally distributed data was analyzed with a one-way analysis of variance (ANOVA). The subsequent post hoc tests for between-group differences were conducted using Tukey's honest significant difference (HSD) test. Comparison of continuous notnormally distributed data were analyzed by means of a Mann-Whitney (M-W) U-test and a Kruskal-Wallis (K-W) ANOVA. The subsequent post hoc tests were conducted by means of

multiple comparisons of mean ranks ${ }^{18}$. Paired continuous data were evaluated using a 
Wilcoxon's Matched Pair test. Analyses of correlations between continuous data were conducted by means of Spearman Rank-order Correlation tests.

Data that were measured repeatedly on more than two occasions were analyzed by means of a repeated measures ANOVA. To ensure that the assumptions of a repeated measures ANOVA were met, the assessment of normal distribution was performed using a normal probability (Q-Q) plot and the homogeneity of variance was assessed by the Mauchly sphericity test. If the sphericity was violated and epsilon was $<0.75$, adjustments of the within subjects factor were carried out with the Greenhouse-Geisser correction method. Post hoc tests for between-groups comparisons were conducted using Tukey's HSD tests.

The significance level was set at $p<0.05$ (two-sided testing). All analyses were carried out according to intention-to-treat principles.

\section{Power analysis}

Sample size estimation was based on the putative outcomes in the EQ-5D index and duration of sick leave. With the supposition that a minimum clinically important difference in the EQ5D index between groups was 0.10 and the SD of the EQ-5D index was 0.20 (obtained from our previous study on benign hysterectomy) ${ }^{10}$ each of the four intervention groups should consist of 91 women included $10 \%$ withdrawals, in order to reach significance at a $5 \%$ level with a power of $90 \%$.

Concerning sick leave, we have previously demonstrated a difference in sick leave of nine days ( $\mathrm{SD}=13$ days) after benign hysterectomy ${ }^{19}$ between women with high and low stress coping capacity. Thus, based on the assumptions that a minimum clinically important difference in sick leave was at least five days between the groups, and the withdrawal rate in the study was $10 \%$, each of the four intervention groups should consist of 150 women in order to achieve significance at a $5 \%$ level with a power of $90 \%$. Consequently, the 
POSTHYSTREC trial was designed to enroll 600 women with an estimated recruitment period of four years.

The study inclusion period spanned more than five years. Due to a lower recruiting rate during the last half year, the inclusion was terminated prematurely when 525 women were enrolled. A post hoc power analysis revealed that this number would ensure a power of at least $80 \%$ instead of the $90 \%$ originally planned.

\section{Missing data}

To determine differences between groups requires data on all occasions of repeated measurements. Thus, missing data in the questionnaires for a specific question were replaced by the truncated mean value of that question for the specific intervention group. The number of missing cells in the EQ-5D 3L made up 5\% in total on all occasions, and in the SF-36, $0.31 \%$ and $1.04 \%$ of the missing cells were substituted in the baseline and six-week form, respectively. 


\section{Results}

The selection of women in the study is presented in the CONSORT flow-chart (Figure 3). In total, 487 women completed the study and constituted the study population. The demographic, medical and clinical data of the groups are presented in Table 1. Analyses of the preoperative and postoperative data showed no statistically significant differences between the intervention groups except for co-morbidity concerning mental illness (comprising mainly mild to moderate anxiety and depressive disorders) that presented in $19.2 \%, 6.6 \%, 16.0 \%$ and $11.7 \%$ of Groups A, B, C and D, respectively.

\section{Effect of intervention on recovery of $H R Q O L$}

The baseline EQ-5D-3L health index was similar in the four groups, ( $p=0.93, \mathrm{~K}-\mathrm{W}$ ANOVA). The time-related recovery in EQ-5D-3L health index in the four intervention groups is presented in Figure 4. No significant differences were seen between the four groups, nor was an interaction effect observed. The same applied when adjusted for mental illness that was imbalanced between the groups. The recovery in EQ-5D-3L health index was faster in those without mental illness than in those with mental illness $(p=0.04)$, but this was not influenced by mode of follow-up strategy. The EQ-5D-3L health index increased significantly over time and reached the baseline level after four weeks. After six weeks the EQ-5D-3L health index levels were significantly higher than the baseline levels in all four groups (all four p's $<0.0001)$. Although the recovery in EQ-5D-3L health index was significantly faster in vaginal hysterectomy $(\mathrm{VH})$ than in the abdominal hysterectomy variants $(\mathrm{p}<0.0001$ for $\mathrm{VH}$ versus total abdominal hysterectomy and $\mathrm{p}<0.001$ for $\mathrm{VH}$ versus subtotal abdominal hysterectomy, respectively) when adjusted for follow-up models, and faster in bleeding and dysplasia as indication for hysterectomy than in myoma uteri only as indication $(p=0.02$ and $\mathrm{p}=0.02$, respectively), this was not attributed to a significant effect of the follow-up models (data not shown). Recovery of Qol, as measured by the EQ-5D-3L index, was not associated 
with age, age groups, BMI or BMI groups or any of the other factors parity, smoking, employment, physical work load, comorbidity with cardio-vascular disease och chronic pain disorder, previous laparotomy, ASA classification or mode of anesthesia when adjusted for follow-up models (data not shown).

None of the SF-36 subscale scores differed significantly at baseline and six weeks postoperatively (Table 2). The recovery in scores is shown in Table 3 . No statistically significant differences were observed between the groups in any of the subscale scores. The recovery in the subscales within each group varied. It seemed that the "physical" recovery, i.e. the PCS, had not reached the baseline level, statistically significantly, after six weeks for any of the four groups. Contrarily, the "mental" recovery, i.e. the MCS, had achieved baseline levels within each group at the six-week assessment.

\section{Sick leave}

The duration of sick leave was in Group A ( $\mathrm{n}=101)$ mean (SD) 26.8 (10.4) days, in Group B $(\mathrm{n}=112) 28.1$ (10.7) days, in Group C ( $\mathrm{n}=105) 28.0$ (10.0) days, and in Group D ( $\mathrm{n}=102) 26.9$ (10.8) days. No significant differences were seen between the groups (one-way ANOVA; $\mathrm{p}=0.71)$.

\section{Unplanned telephone contact and visits to health care facilities.}

As shown in Table 4, the women in the intervention Group D initiated unplanned telephone contact with a health care facility significantly less often (lower by about $30 \%$ ) than the women in the other three groups. This happened in spite of the fact that the number of women who developed complications postoperatively within six weeks after surgery and the grade of the complication according to the Clavien-Dindo classification did not differ significantly between the intervention groups.

The significantly lower frequency of unplanned telephone contact in Group D remained even when looking at unplanned telephone contact that was not followed by an unplanned visit. In 
contrast, the unplanned visits, irrespective of whether they were preceded by an unplanned telephone contact or not, did not differ between the groups.

Associations between recovery of HRQoL and duration of sick leave.

The repeated measures ANOVA revealed that the recovery as determined by EQ-5D-3L health index when adjusted for follow-up models was significantly associated with duration of sick leave $(\mathrm{p}<0.0001)$ even when adjusted for body mass index, smoking, physical workload, mode of hysterectomy and postoperative complications, which were the five factors that in univariate analyses were found to be independent predictors for recovery of the EQ-5D-3L (data not shown). Likewise, recovery measured by the SF-36's PCS (Spearman's rho = 0.13; $\mathrm{p}<0.01$ ), but not the SF-36's MCS (Spearman's rho =-0.05; $\mathrm{p}=0.29$ ) correlated with duration of sick leave. 


\section{Discussion}

Although the study showed that the structured coaching program used in this study or repeated weekly planned phone contact with nurses neither accelerated the recovery in HRQoL nor reduced the duration of sick leave after benign hysterectomy compared with no planned telephone contact, the coaching program seemed to reduce the number of unplanned contacts with the health care services. Duration of sick leave was significantly associated with recovery of HRQoL as measured by EQ-5D-3L health index and SF-36' PCS, but not with SF-36's MCS.

The study did not show any benefit of the coaching program or the nurses' phone contact on recovery in HRQoL or sick leave. Despite this, the coaching program seemed to reduce the number of unplanned telephone contacts, indicating a positive effect of the coaching sessions. The literature is very limited as regards quantitative studies on postoperative follow-up contact with the purpose of accelerating recovery. To the best of our knowledge, this study is the first RCT that has investigated the impact of follow-up strategies on recovery after hysterectomy. Several qualitative studies have indicated that patients strongly believe that their recovery will benefit from follow-up contact with nurses ${ }^{6,20-22}$. Unfortunately, until now, these hypothesis-generating studies have not been followed up by clinical trials in order to verify the effect of such efforts. Thus, the results of the present study can be seen as a rejection of the hypotheses derived from these qualitative studies.

Psychological preparation with either procedural information, sensory information, cognitive intervention, relaxation, hypnosis or emotion-focused intervention has been indicated to be beneficial for the postoperative outcomes concerning postoperative pain, behavioral recovery, negative effects and length of hospital stay, and is found unlikely to be harmful ${ }^{23}$. However, the authors of that report concluded that the strength of evidence was insufficient to reach firm conclusions on the role of psychological preparation in surgery. Our 
experimental intervention can be seen as an intensive coaching strategy of managing postoperative symptoms that might be experienced differently in certain sensitive subgroups of patients. Thus, it would be plausible that such an intervention should also allow accelerated recovery in the experimental intervention group compared with the other three groups. Even though we failed to demonstrate such an effect even in subgroups, we showed that unplanned telephone contact occurred significantly less often in the coaching group. This might imply that the coaching had prepared the patient better to cope reasonably with the postoperative symptoms and disability. Based on these indications we believe that our findings are valid and generalizable to societies with similar socio-demographic and health care facilities.

A possible explanation of why we failed to demonstrate an accelerated recovery in the coaching group might be the systematic use of ERAS, which provides thorough preoperative information about the postoperative recovery. Although this information was given broadly, it might possibly have affected the women in all four groups sufficiently to accelerate the recovery and thus reduced the potential benefit of structured coaching.

Sick leave after surgery can be seen as a surrogate measure of recovery. Several factors, other than surgical pathophysiological factors, might influence the duration of sick leave after surgery ${ }^{24-27}$. This study showed significant associations between duration of sick leave and recovery of HRQoL, both in the short and longer terms. However, long-term it was seen for physical recovery whereas no association was seen in mental recovery. These results might indicate that the duration of sick leave in a Swedish setting can also been seen as a valid measure for recovery.

The randomized design and the large number of participants are major strengths of the study. Another important strength is the coaching program and the education of the research nurses, with repeated sessions and support. The coaching program was developed by a clinically skilled behavior therapist (GS), experienced in cognitive behavior therapy (CBT). 
The research nurses received standardized education in the coaching technique given by the behavior therapist prior to the start of the study. The education session was repeated at the annual trial meetings during the course of the study and the nurses had access to support from the behavior therapist on request if necessary. CBT has been proved to facilitate recovery and improve quality of life after psychological and physical trauma. The coaching program in the present study was based on elements derived from CBT and consequently, theoretically, could be effective for enhancing postoperative recovery.

Other strengths are the allocation into four groups, of which three reflected the commonly used models of postoperative follow-up and the fourth represented the experimental intervention. In order to demonstrate short-term and long-term variations in HRQoL the EQ-5D and SF-36 forms were used.

The study has some limitations. The study terminated prematurely. However, it still met the power requirements usually requested for clinical trials i.e. $80 \%$. We therefore believe that the results and interpretation in our study remain reliable. Some may see the lack of specific patient-oriented questions in this trial as a limitation as the standardized questionnaires might not have reflected outcomes that were important to the patient or related to the intervention itself. However, such consideration may raise concerns on the validity of using validated HRQoL forms as outcome measures in clinical trials.

The single-blinding may be seen as a strength because it reduces placebo effects ${ }^{28,29}$, but is also a limitation since the research nurses were at risk of mixing up the content of the oriented coaching program (Group D) and the standard postoperative counselling (Group C). However, the research nurses were carefully instructed on several occasions to be aware of this and actively worked to avoid admixing the interventions. We therefore believe that the problems with admixing of the interventions were largely avoided. For practical reasons, 
double blinding was not possible due to the restricted access to research nurses who accomplished the follow-up contact interventions.

The oriented coaching program used in this study has not been strictly validated. Neither has the patient's perceived personal value of the intervention strategies been evaluated which may influence the interpretation of the results. However, we believe that the coaching program was founded on a sound theoretical and practical clinical basis that made it useful for its purpose. The inspiration of the coaching program was based on elements from CBT. CBT is well researched and clinically effective, and is used in many clinical situations. On the other hand, a strict CBT program requires time, education and resources and may therefore per se be less useful for handling the short-term recovery period after hysterectomy. The use of educated research nurses for coaching might be considered sufficient to improve health outcomes ${ }^{30}$ and thus may help to achieve an accelerated short-term recovery after hysterectomy.

Well-designed RCTs are needed to evaluate the clinical effect of measures to improve postoperative recovery. Such studies are also urgently warranted in order to study the hypotheses derived from qualitative studies on patients' demands, expectations and requests to decide whether these preferences de facto give measurable effects on clinical outcomes and to ensure that the health care resources are used optimally.

\section{Conclusion}

Compared with no follow-up contact, neither the oriented coaching performed at the planned, weekly telephone follow-up contact by nurses, nor the other modes of follow-up contact that was used in this trial seemed to accelerate the postoperative recovery in HRQoL or to reduce the duration of sick leave after benign hysterectomy in an ERAS setting. However, the coaching model seemed to reduce unplanned telephone contact postoperatively, and thus reduced the workload of the health care facilities. This information is important and a health 
economy evaluation should be considered before a postoperative coaching model is introduced for women undergoing hysterectomy on benign indications. 


\section{Acknowledgement}

The authors thank all women who participated in this trial. We are deeply grateful for the committed work conducted by all in the POSTHYSTREC study group, in particular by the research nurses. Special thanks for invaluable work go to Åsa Rydmark Kersley, RN, MSc and to the external monitor Lotta Lindh Åstrand, RN, PhD, for their meticulous work.

\section{The POSTHYSTREC Study Group}

The POSTHYSTREC Study Group consisted of members from five hospitals in the South east region of Sweden.

Linköping University Hospital: Preben Kjølhede, MD, PhD, Gunilla Sydsjö, PhD, Ninnie Borendal Wodlin, MD, PhD, Lena Nilsson, MD, PhD, Gulnara Kassymova, MD, Peter Lukas, MD, Petra Langström, RN, Pernilla Nilsson, RN, Linda Shosholli, RN, Sofia Bergström, RN, and Åsa Rydmark Kersley, RN, MSc.

Vrinnevi Hospital, Norrköping: Leif Hidmark, MD, Anders Bolling, MD, Kristina Ekman, RNW, and Karin Granberg-Karlsson, RNW

Ryhov Hospital, Jönköping: Laila Falknäs, MD, Maria Häggström, MD, Ewa Hermansson RNW

Eksjö Highland Hospital: Tomaz Stypa, MD, PhD, Linda Myllimäki, MD, Iréne Johannesson, RNW, and Martina Ekeroth Wikander, RNW.

Värnamo Hospital: Christina Gunnervik, MD, Fatima Johansson, MD Magnus Trofast, MD, Mari-Ann Andersson, RNW, and Carita Jacobsson, RN. 


\section{Authorship confirmation statement}

The study was conceptualized by PK, GS, LN and NBW who also wrote the research protocol. The study was conducted by PK, LN, GK and NWB. The main author is GK. Analyses of data were performed by PK and GK. All authors contributed to revision of the manuscript and all approved the final version of the manuscript 


\section{Authors' disclosure statements}

None of the authors has competing financial interests to declare. 


\section{Funding}

The study was supported by grants from the Medical Research Council of Southeast Sweden (grant numbers FORSS-155141; FORSS-222211; FORSS-308441, and FORSS-387761), and Futurum - the Academy of Health and Care, Region Jönköping Council (grant numbers FUTURUM-487481, and FUTURUM 579171). In addition, Region Östergötland Council and Linköping University contributed with unrestricted grants. 


\section{References}

1. Hammer A, Rositch AF, Kahlert J,Gravitt PE, Blaakär J, Søgård M. Global epidemiology of hysterectomy: possible impact on gynecological cancer rates. Am J Obstet Gynecol. 2015;213(1):23-9.

2. Nelson G, Altman AD, Nick A, et al. Guidelines for postoperative care in gynecologic/oncology surgery: Enhanced Recovery After Surgery (ERAS(®)) Society recommendations--Part II. Gynecol Oncol. 2016;140(2):323-32.

3. Nelson G, Altman AD, Nick A, et al. Guidelines for pre- and intra-operative care in gynecologic/oncology surgery: Enhanced Recovery After Surgery (ERAS(®)) Society recommendations--Part I. Gynecol Oncol. 2016;140(2):313-22.

4. Mavros MN, Athanasiou S, Gkegkes ID, Polyzoz KA, Peppas G, Falagas ME. Do psychological variables affect early surgical recovery? PLoS One. 2011;6(5):e20306.

5. Gillis C, Gill M, Marlett N, et al. Patients as partners in Enhanced Recovery After Surgery: A qualitative patient-led study. BMJ Open. 2017;7(6):e017002.

6. Sibbern T, Bull Sellevold V, Steindal SA, Dale C, Watt-Watson J, Dihle A. Patients' experiences of enhanced recovery after surgery: a systematic review of qualitative studies. J Clin Nurs. 2017;26(9-10):1172-88.

7. Cuijpers P, Sijbrandij M, Koole S, Huibers M, Berking M, Andersson G. Psychological treatment of generalized anxiety disorder: a meta-analysis. Clin Psychol Rev. $2014 ; 34(2): 130-40$.

8. Bowyer A, Royse C. The importance of postoperative quality of recovery: influences, assessment, and clinical and prognostic implications. Can J Anaesth. 2016;63(2):17683. 
9. Bowyer A, Royse CF. The future of postoperative quality of recovery assessment: multidimensional, dichotomous, and directed to individualize care to patients after surgery. Curr Opin Anaesthesiol. 2016;29(6):683-90.

10. Dindo D, Demartines N, Clavien PA. Classification of surgical complications: a new proposal with evaluation in a cohort of 6336 patients and results of a survey. Ann Surg. 2004;240(2):205-13.

11. Simple Interactive Statistical Analysis. Allocation. [Available from: https://www.quantitativeskills.com/sisa/][cited 2020 June 24]

12. EuroQoL Group. EuroQol--a new facility for the measurement of health-related quality of life. Health policy. 1990;16(3):199-208.

13. Burstrom K, Johannesson M, Diderichsen F. Swedish population health-related quality of life results using the EQ-5D. Qual Life Res. 2001;10(7):621-35.

14. McHorney CA, Ware JE, Jr., Lu JF, Sherbourne CD. The MOS 36-item Short-Form Health Survey (SF-36): III. Tests of data quality, scaling assumptions, and reliability across diverse patient groups. Med Care. 1994;32(1):40-66.

15. Sullivan M, Karlsson J, Ware JE, Jr. The Swedish SF-36 Health Survey--I. Evaluation of data quality, scaling assumptions, reliability and construct validity across general populations in Sweden. Soc Sci Med. 1995;41(10):1349-58.

16. The World Medical Association. WMA Declaration of Helsingki - Ethical principles for medical research involving human subjects [Available from: https://www.wma.net/policies-post/wma-declaration-of-helsinki] [cited 2018 July 9].

17. International Council for Harmonisation of Technical Requirements for Pharmaceuticals for Human Use. ICH GCP Good Clinical Practice [Available from: https://www.wma.net/policies-post/wma-declaration-of-helsinki-ethical-principles-formedical-research-involving-human-subjects/ ][cited 2019 Dec 21] 
18. Siegel S, Castellan NJ. Nonparametric statistics for the behavioral sciences (2nd ed): New York: McGraw-Hill.; 1988. p. 213-5.

19. Kjølhede P, Borendal Wodlin N, Nilsson L, Fredrikson M, Wijma K. Impact of stress coping capacity on recovery from abdominal hysterectomy in a fast-track programme: a prospective longitudinal study. BJOG. 2012;119(8):998-1006.

20. Berg K, Arestedt K, Kjellgren K. Postoperative recovery from the perspective of day surgery patients: a phenomenographic study. Int J Nurs Stud. 2013;50(12):1630-8.

21. Mottram A. 'They are marvellous with you whilst you are in but the aftercare is rubbish': a grounded theory study of patients' and their carers' experiences after discharge following day surgery. J Clin Nurs. 2011;20(21-22):3143-51.

22. Renholm M, Souminen T, Turtiainen A-M, Leion-Kilpi H. Continuity of care in ambulatory surgery critical pathways: the patients' perceptions. Medsurg Nurs. 2009;18(3):169-73.

23. Powell R, Scott NW, Manyande A, et al. Psychological preparation and postoperative outcomes for adults undergoing surgery under general anaesthesia. Cochrane Database Syst Rev. 2016(5):CD008646.

24. Bohlin KS, Ankardal M, Nussler E, Lindkvist H, Milsom I. Factors influencing the outcome of surgery for pelvic organ prolapse. Int Urogynecol J. 2018;29(1):81-9.

25. Bohlin KS, Ankardal M, Pedroletti C, Lindkvist H, Milsom I. The influence of the modifiable life-style factors body mass index and smoking on the outcome of midurethral sling procedures for female urinary incontinence. Int Urogynecol J. 2015;26(3):343-51.

26. Reber KC, Konig HH, Hajek A. Obesity and sickness absence: results from a longitudinal nationally representative sample from Germany. BMJ Open. 2018;8(6):e019839. 
27. Weng SF, Ali S, Leonardi-Bee J. Smoking and absence from work: systematic review and meta-analysis of occupational studies. Addiction. 2013;108(2):307-19.

28. Berthelot JM, Le Goff B, Maugars Y. The Hawthorne effect: stronger than the placebo effect? Joint Bone Spine. 2011;78(4):335-6.

29. McCambridge J, Witton J, Elbourne DR. Systematic review of the Hawthorne effect: new concepts are needed to study research participation effects. J Clin Epidemiol. 2014;67(3):267-77.

30. Ross A, Brooks AT, Yang L, Touchton-Leonard K, Raju S, Bevans M. Results of a national survey of certified nurse coaches with implications for advanced practice nurses. J Am Assoc Nurse Pract. 2018;30(5):251-61. 


\section{Figure legends}

Figure 1. Examples of the standardized format questions to Group B and C, asked by the research nurse.

Figure 2. Content of the oriented coaching strategies in guiding and support, influenced by cognitive behavior therapy, used by the research nurse in the follow-up of Group D.

Figure 3. CONSORT flow-chart of participants in the POSTHYSTREC trial.

Figure 4. EQ-5D-3L health index after hysterectomy in the four intervention groups. Plots indicate means and bars $95 \%$ confidence intervals. The repeated measures ANOVA comprises the measurements from Day 0 to Day 42. The results of the repeated measures ANOVA are demonstrated in the table below the graph. * Sphericity violated; p-value corrected according to the Greenhouse-Geisser correction method. 


$\begin{array}{ll}\text { General wellbeing: } & \text { "How have you been since you were discharged?" } \\ \text { Experience of pain: } & \text { "Have the pain been manageable?" } \\ \text { Nutrition: } & \text { "Have you been able to eat and drink?" } \\ \text { Elimination: } & \begin{array}{l}\text { Does it feel like normal when you urinate"? "Have you passed gas/had a } \\ \text { bowel movement?" }\end{array} \\ \begin{array}{l}\text { Surgical wound/ } \\ \text { Dressing of the wound: }\end{array} & \text { "Have you taken a look at the wound and "how does it look"? "Is the }\end{array}$

Figure 1. Examples of the standardized format questions to Group B and C, asked by the research nurse. 
- $\quad$ Standardized evaluation of the well-being of the patient, according to the standardized format described for Group B and C.

- Discussion on how the patient is doing regarding physical pain, worries, and sleep. Provide the patient with strategies to increase the well-being, e.g. rest, relaxation techniques, and pain relief alternatives.

- Balance thoughts of worry - Restructure negative thoughts and provide alternative interpretations.

- Inform the patient on how interpretations and how we perceive events are affected when we are tired or in pain.

- Validate improvements, i.e. enforce positive events and events that the patient perceives as positive.

- Make use of strategies that the woman usually uses when experiencing pain, worry, or sleep problems.

Figure 2. Content of the oriented coaching strategies in guiding and support, influenced by cognitive behavior therapy, used by the research nurse in the follow-up of Group D. 


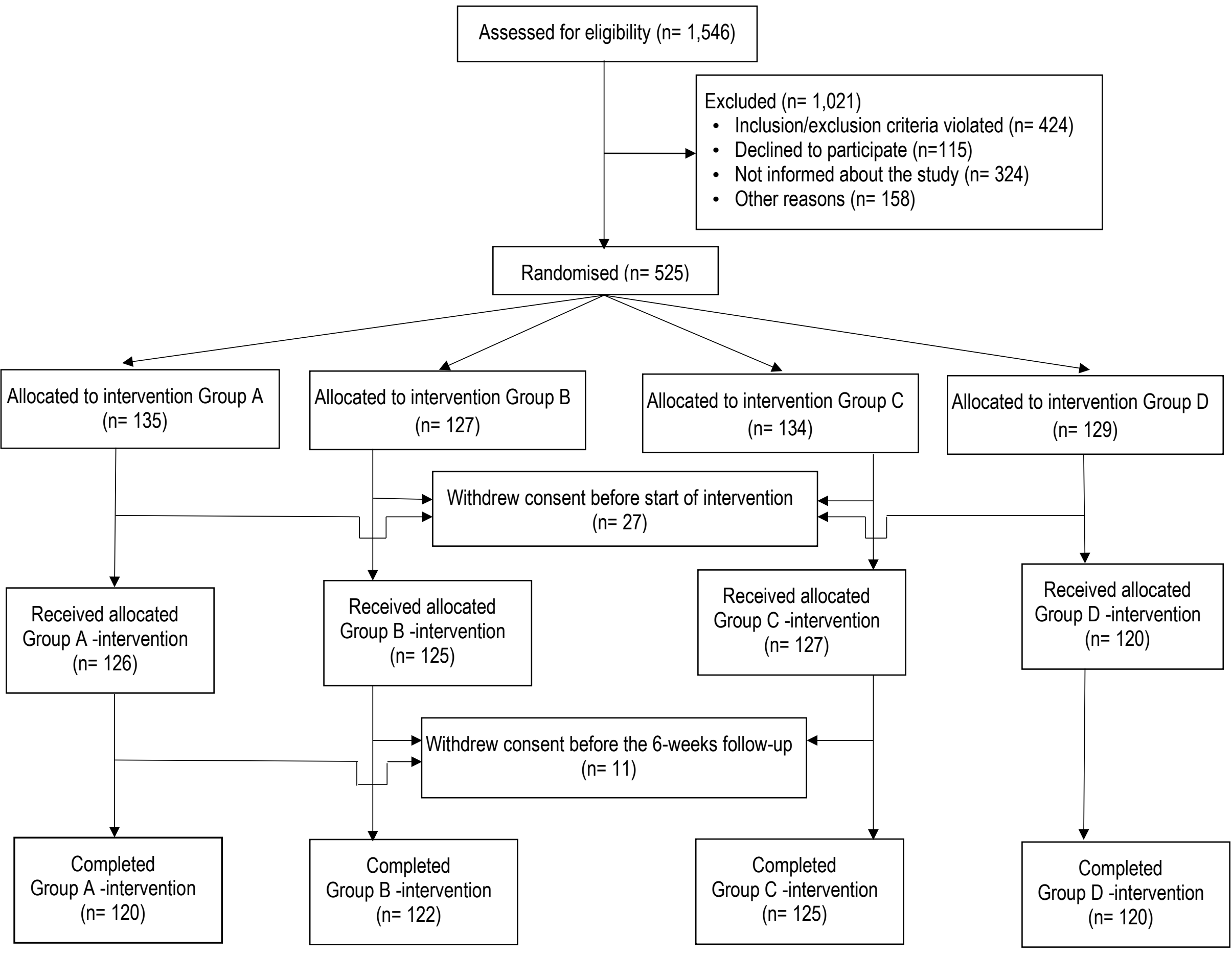

Figure 3. CONSORT flow-chart of participants in the POSTHYSTREC trial. 


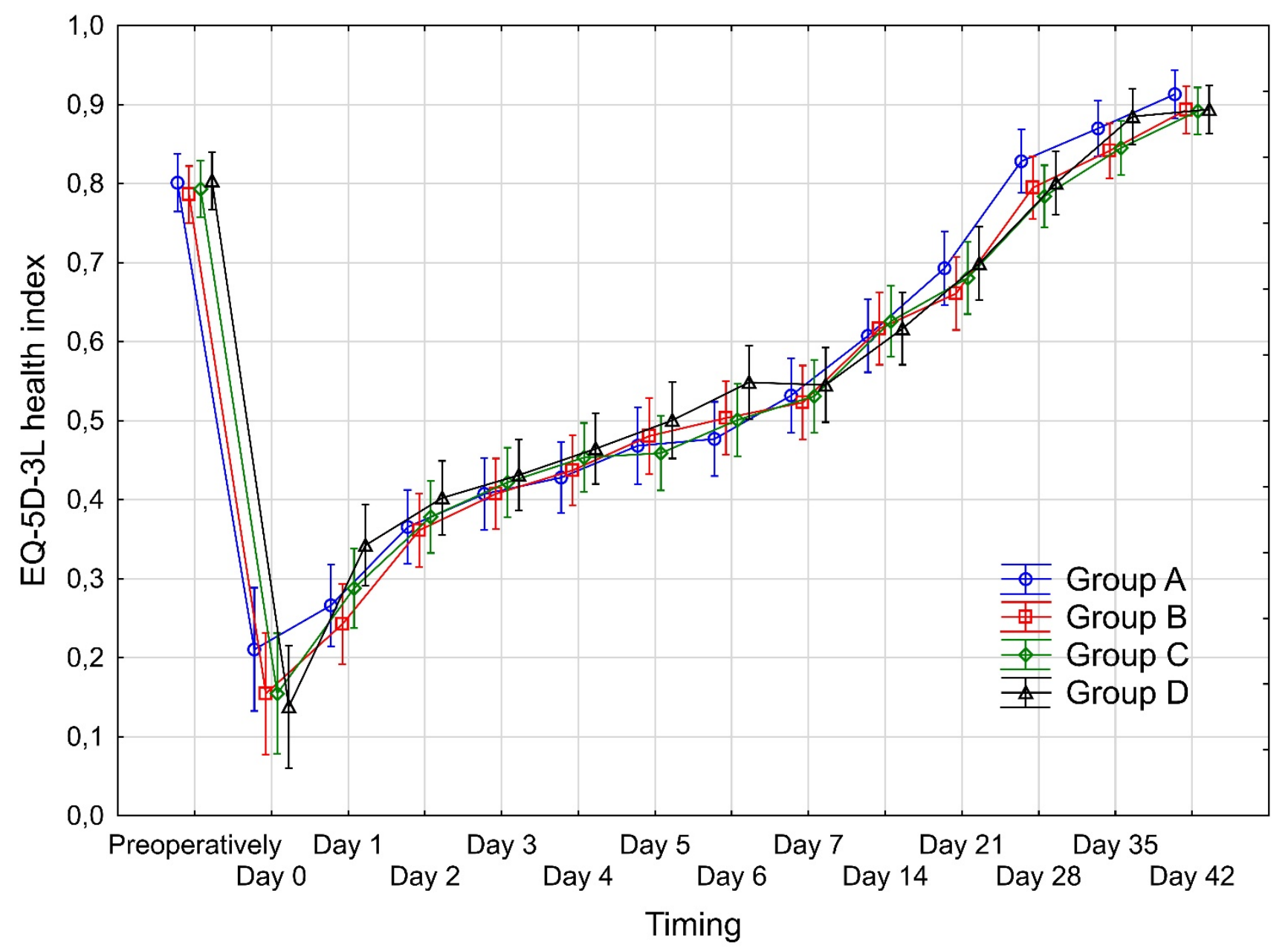

Repeated measures ANOVA

\begin{tabular}{llccc} 
& & Effect between groups & Effect over time & Interaction effect \\
\hline EQ-5D-3L health index & Crude & 0.71 & $<0.0001^{*}$ & $0.34^{*}$ \\
\hline & Adjusted $\dagger$ & 0.66 & $<0.0001^{*}$ & $0.37^{*}$ \\
\hline
\end{tabular}

Figure 4. EQ-5D-3L health index after hysterectomy in the four intervention groups. Plots indicate means and bars $95 \%$ confidence intervals. The repeated measures ANOVA comprises the measurements from Day 0 to Day 42. The results of the repeated measures ANOVA are demonstrated in the table below the graph. $\uparrow$ Adjusted for mental illness. * Sphericity violated; p-value corrected according to the Greenhouse-Geisser correction method. 
Table 1. Demographic and clinical descriptive data of 487 women undergoing benign hysterectomy subdivided after intervention group.

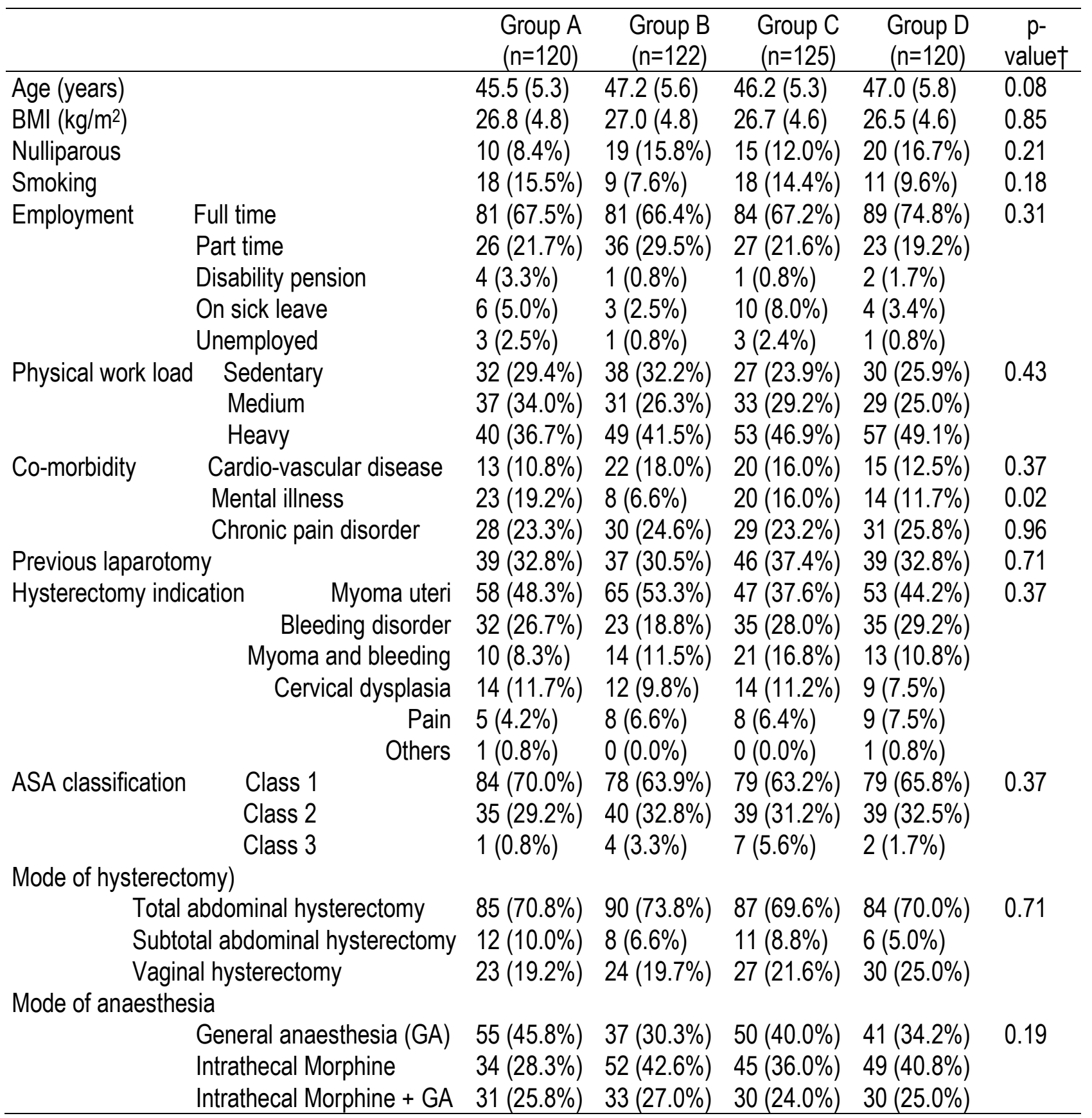

Figures denote mean and (one standard deviation) or number of women and (per cent).

$\uparrow$ One-way ANOVA used for continuous data and Pearson's chi-squared tests for nominal data. 
Table 2. Baseline and 6-weeks postoperative scores of subscales of SF-36.

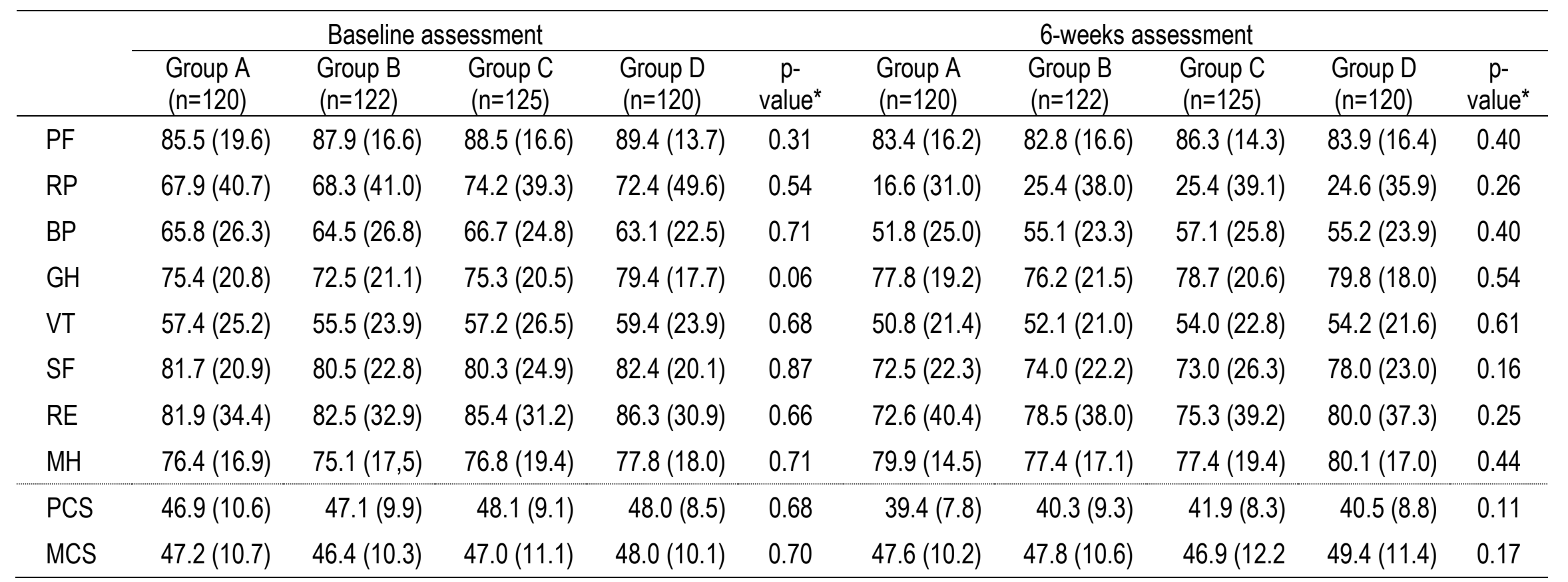

Figures denote mean and (one standard deviation).

* Kruskal-Wallis analysis of variance.

$\mathrm{PF}=$ Physical functioning. $\mathrm{RP}=$ Role limitation physical. $\mathrm{BP}=$ Bodily pain. $\mathrm{GH}=$ General health; VT $=\mathrm{Vitality} . \mathrm{SF}=\mathrm{Social}$ functioning. $\mathrm{RE}=$ Role limitation emotional. $\mathrm{MH}=$ Mental health. $\mathrm{PCS}=$ Physical component summary. $\mathrm{MCS}=$ Mental component summary. 
Table 3. Changes in SF-36 subscale scores within and between groups at baseline and the 6-weeks postoperative assessment.

\begin{tabular}{|c|c|c|c|c|c|c|c|c|c|}
\hline & \multicolumn{4}{|c|}{$\begin{array}{l}\text { Difference in subscale scores between 6-weeks } \\
\text { postoperatively and baseline }\end{array}$} & \multirow[t]{2}{*}{$\begin{array}{l}\text { Between-group analyses } \\
\text { p-value }\end{array}$} & \multicolumn{4}{|c|}{$\begin{array}{l}\text { Within-group analyses* } \\
\text { p-value }\end{array}$} \\
\hline & Group A & Group B & Group C & Group D & & Group A & Group B & Group C & Group D \\
\hline PF & $-2.1(20.9)$ & $-5.2(15.6)$ & $-2.2(17.5)$ & $-5.5(17.2)$ & 0.50 & 0.04 & $<0.001$ & 0.03 & $<0.001$ \\
\hline $\mathrm{RP}$ & $-51.3(47.0)$ & $-42.9(50.8)$ & $-48.8(50.8)$ & $-47.9(53.5)$ & 0.53 & $<0.0001$ & $<0.0001$ & $<0.0001$ & $<0.0001$ \\
\hline $\mathrm{BP}$ & $-14.0(27.8)$ & $-9.3(31.7)$ & $-9.5(30.9)$ & $-7.9(27.7)$ & 0.39 & $<0.0001$ & $<0.01$ & $<0.01$ & $<0.01$ \\
\hline $\mathrm{GH}$ & $2.5(16.8)$ & $3.7(15.5)$ & $3.4(17.0)$ & $0.4(14.2)$ & 0.15 & 0.09 & $<0.01$ & 0.01 & 0.78 \\
\hline VT & $-6.6(23.8)$ & $-3.3(21.1)$ & $-3.2(27.3)$ & $-5.2(26.7)$ & 0.65 & $<0.01$ & 0.08 & 0.14 & 0.02 \\
\hline SF & $-9.2(25.3)$ & $-6.5(23.6)$ & $-7.3(32.8)$ & $-4.4(24.0)$ & 0.47 & $<0.0001$ & $<0.01$ & 0.01 & 0.08 \\
\hline RE & $-9.3(46.2)$ & $-4.0(39.0)$ & $-10.0(44.8)$ & $-6.3(42.1)$ & 0.63 & 0.04 & 0.38 & 0.02 & 0.10 \\
\hline $\mathrm{MH}$ & $3.4(17.1)$ & $2.3(16.4)$ & $0.6(19-0)$ & $2.3(18.7)$ & 0.89 & 0.03 & 0.05 & 0.40 & 0.14 \\
\hline PCS & $-7.5(10.7)$ & $-6.8(10.2)$ & $-6.2(10.7)$ & $-7.5(10.5)$ & 0.74 & $<0.0001$ & $<0.0001$ & $<0.0001$ & $<0.0001$ \\
\hline MCS & $0.4(11.8)$ & $1.4(9.7)$ & $-0.1(13.3)$ & $1.4(11.9)$ & 0.52 & 0.81 & 0.03 & 0.40 & 0.05 \\
\hline
\end{tabular}

Figures denote mean difference (one standard deviation) in the subscale score between the 6-weeks postoperative assessment and the baseline assessment. A negative value indicates that the group has not recovered to the baseline level.

$\mathrm{PF}=$ Physical functioning. $\mathrm{RP}=$ Role limitation physical. $\mathrm{BP}=$ Bodily pain. $\mathrm{GH}=$ General health; $\mathrm{VT}=\mathrm{Vitality} . \mathrm{SF}=\mathrm{Social}$ functioning.

$\mathrm{RE}=$ Role limitation emotional. $\mathrm{MH}=$ Mental health. $\mathrm{PCS}=$ Physical component summary. $\mathrm{MCS}=$ Mental component summary.

\# Kruskal-Wallis ANOVA

* Wilcoxon Matched Pair tests. 
Table 4. Postoperative complications and unplanned telephone contacts or visits in health care facilities after discharge from surgery until six weeks postoperatively in relation to intervention group.

\begin{tabular}{|c|c|c|c|c|c|c|}
\hline & & $\begin{array}{l}\text { Group A } \\
(n=120)\end{array}$ & $\begin{array}{l}\text { Group B } \\
(n=122)\end{array}$ & $\begin{array}{l}\text { Group C } \\
(n=125)\end{array}$ & $\begin{array}{l}\text { Group D } \\
(n=120)\end{array}$ & $\mathrm{p}$-value \\
\hline \multicolumn{2}{|l|}{ Complications postoperatively } & $37(30.8 \%)$ & $40(32.8 \%)$ & $44(35.2 \%)$ & $28(23.3 \%)$ & $0.21^{*}$ \\
\hline \multirow[t]{3}{*}{ Clavien-Dindo classification of complicationsł: } & Grade I & $17(14.2 \%)$ & $15(12.3 \%)$ & $16(12.8 \%)$ & $9(7.5 \%)$ & $0.64 \dagger$ \\
\hline & Grade II & $17(14.2 \%)$ & $19(15.6 \%)$ & $24(19.2 \%)$ & $16(13.3 \%)$ & \\
\hline & Grade III & $3(2.5 \%)$ & $6(4.9 \%)$ & $4(3.2 \%)$ & $3(2.5 \%)$ & \\
\hline \multicolumn{2}{|l|}{ Unplanned telephone contacts \# } & $57(47.5 \%)$ & $65(53.3 \%)$ & $62(49.6 \%)$ & $40(33.3 \%)$ & $0.01^{*}$ \\
\hline \multicolumn{2}{|c|}{ Unplanned telephone contacts without succeeding visit } & $22(18.3 \%)$ & $26(21.7 \%)$ & $19(15.2 \%)$ & $13(10.8 \%)$ & $<0.05^{*}$ \\
\hline \multicolumn{2}{|l|}{ Unplanned visits \# } & $52(43.3 \%)$ & $50(41.0 \%)$ & $62(49.6 \%)$ & $39(32.5 \%)$ & $0.06^{*}$ \\
\hline \multicolumn{2}{|c|}{ Unplanned visit preceded by unplanned telephone contact } & $35(29.2 \%)$ & $39(32.0 \%)$ & $43(34.4 \%)$ & $27(22.5 \%)$ & $0.11^{*}$ \\
\hline \multicolumn{2}{|c|}{ Unplanned visit without preceded unplanned telephone contact } & $17(14.2 \%)$ & $11(9.0 \%)$ & $19(15.2 \%)$ & $12(10.0 \%)$ & $0.20^{*}$ \\
\hline
\end{tabular}

Figures denote number of women and (per cent).

$\ddagger$ Clavien-Dindo contracted classification of postoperative complications.

* Pearson's chi-squared test $(\mathrm{df}=3)$.

$\dagger$ Pearson's chi-squared test $(\mathrm{df}=6)$

\# Patient initiated telephone contact or visit in health care facilities not planned in advance between discharge after surgery and the 6-weeks follow-up visit 
Supplemental Table S 1. Recommended standardized regimes in the ERAS protocol.

\section{Preoperatively}

Information

Premedication

Intraoperatively

Anesthesia

Parenteral fluids

Local

anesthesia

Prevention

Bladder catheter

Surgery

\section{Postoperatively}

Post-anesthesia

care unit

(PACU)

Gynecological

ward.

After discharge

from hospital
Oral and written information given and repeated concerning pre- and peri-operative care, management of pain and PONV, early postoperative ambulation, enteral nutrition and discharge criteria. Smoking cessation recommended.

Fasting for six hours.

Clear fluids orally allowed until two hours before surgery.

$1995 \mathrm{mg}$ paracetamol orally were given one hour before surgery.

Recommended: Intrathecal anesthesia with bupivacaine $20 \mathrm{mg}(5 \mathrm{mg} / \mathrm{ml})+$ morphine $0.2 \mathrm{mg}(0.4$ $\mathrm{mg} / \mathrm{ml}$ ) combined with propofol sedation. Alternatively combined with: General anesthesia. Induction with fentanyl 100-200 $\mathrm{\mu g}$ and propofol, intubation facilitated by rocuronium $0.6 \mathrm{mg} / \mathrm{kg}$ and maintenance with propofol infusion $6-10 \mathrm{mg} / \mathrm{kg}^{*} \mathrm{~h}$. Fentanyl and rocuronium iterated as needed. An orogastric tube was applied after anesthesia and removed before waking the patient. Morphine 3-10 mg iv approximately 20 min before end of surgery

A restricted regimen mainly based on crystalloids and aimed at $25 \mathrm{ml} / \mathrm{kg}$ and day. Ended after 24 hours provided vital signs were stable.

A vasoactive agent, preferably ephedrine or phenylephrine, was given if systolic blood pressure decreased $>30 \%$ from the baseline.

$100 \mathrm{mg}$ bupivacaine $(2.5 \mathrm{mg} / \mathrm{ml})$ were injected paracervically at start of vaginal hysterectomy or injected subcutaneously and pre-fascially in abdominal wound at conclusion of abdominal hysterectomy.

Preemptive antiemetic with acupressure wrist bands, applied preoperatively and maintained through hospital stay.

Antibiotic and antithrombotic prophylaxes were administered according to departmental routines.

Maintenance of normothermia by active air-forced warming or hot blankets.

Transurethral catheter was inserted before start of surgery. Catheter was left until next morning. Applying the principles of the technique of minimal invasive surgery, independent of mode of surgery.

Pain management was initiated orally with paracetamol and diclofenac. Additional pain management with iv morphine was offered if VAS (visual analog scale) $>3$. If insufficiently pain relieved, epidural analgesia or patient-controlled analgesia with morphine could be offered as judged by the attending anesthetist.

The woman was permitted to drink. Ambulation was actively encouraged.

Rescue antiemetic treatment when needed was given with droperidol and/or 5-HT3 receptor antagonist.

The woman was discharged to the gynecological ward when vital signs were stable.

Monitoring of hemodynamic and respiratory stability, sedation, pain, nausea and pruritus was conducted once every hour during first 12 hours postoperatively, then once every third hour for another 12 hours.

Pain management orally was continued with $1995 \mathrm{mg}$ paracetamol x 3 and $50 \mathrm{mg}$ diclofenac $\times 3$ daily. Additional pain relief was offered if VAS $>3$, or on patient request. Opioids avoided if possible. Rescue antiemetic treatment as in PACU.

The woman was encouraged to drink and eat as soon as possible. Mobilization was actively encouraged.

Standardized criteria of discharge: woman was mobilized, tolerated normal diet, had sufficient pain control with oral analgesia (VAS $\leq 4$ ), had no signs of mechanical bowel obstruction and had preferable spontaneous voiding with residual urine $\leq 150 \mathrm{ml}$; otherwise, a transurethral catheter was left at discharge.

Pain management continued orally with $1995 \mathrm{mg} \times 3$ paracetamol and $50 \mathrm{mg} \times 3$ diclofenac daily. If NSAID were contraindicated combined codeine/paracetamol $30 \mathrm{mg} / 500 \mathrm{mg} 2 \times 4$ or tramadol $50 \mathrm{mg} \mathrm{x}$ 4 daily were given. A package of 6 tablets of oxycodone (10 mg twice daily) was given to the woman, if necessary. The woman decides duration of treatment with analgesics. 
